\title{
Prevalence of Restless Legs Syndrome in Patients Treated with Peritoneal Dialysis: Clinical and Biochemical Characteristics among Patients with and without Restless Legs Syndrome
}

\author{
Selda Korkmaz ${ }^{1}$, Bulent Tokgoz ${ }^{2}$, Sevda Ismailogullari ${ }^{3}$, Ismail Kocyigit ${ }^{2}$, Merva Kocyigit ${ }^{3}$, \\ Ozgur Berkay Aksu', Murat Aksu ${ }^{3}$ \\ ${ }^{1}$ Department of Neurology, Acibadem Hospital, Kayseri, Turkey \\ ${ }^{2}$ Department of Nephrology, Erciyes University Medical School, Kayseri, Turkey \\ ${ }^{3}$ Department of Neurology, Erciyes University Medical School, Kayseri, Turkey \\ ${ }^{4}$ Medical Faculty, Near East University, Nicosia, Cyprus \\ Email: aksu@erciyes.edu.tr
}

Received December 21, 2012; revised February 10, 2013; accepted March 12, 2013

\begin{abstract}
Introduction and Aims: This is a prospective study identifying prevalence of Restless Legs Syndrome (RLS) in patients ongoing peritoneal dialysis (PD) due to end stage renal disease (ESRD) and comparing clinical and biochemical characteristics among patients with and without RLS. Methods: Two hundred ESRD patients who received PD at least six months assessed by neurologist and nephrologist with regards to presence of RLS. Also, clinical and biochemical features of these patients are determined. One hundred and forty four patients were excluded from study because they had other secondary causes of RLS except for ESRD. Results: Thirteen of 56 patients (23.2\%) had RLS. The use of vitamin B + folic acid supplements was significantly lower in patients with RLS than in those without RLS (69.2\% vs $97.7 \% ; \mathrm{p}=0.008)$. There was no significant difference between patient groups with and without RLS in terms of age, gender, body mass index, cause of ESRD, peritoneal membrane transport characteristic, smoking, consuming alcohol, use of erythropoietin, duration of PD, hemoglobin, serum calcium, serum phosphorus, serum albumin, levels of serum iron, total iron binding capacity, ferritin, folic acid and vitamin B12, transferrin saturation, weekly $\mathrm{Kt} / \mathrm{V}$ urea value, and amount of residual urine volume ( $p>0.05$ ). Conclusion: RLS is more common among PD patients than general population. Although essential cause is not exactly known, use of folic acid and vitamin B complex decrease the RLS prevalence in this particular patient group.
\end{abstract}

Keywords: Peritoneal Dialysis; Restless Legs Syndrome; Renal Failure

\section{Introduction}

Restless legs syndrome (RLS) is the most prevalent sensorimotor disorder during sleep. The prevalence of RLS is estimated to be $5 \%-15 \%$ in general [1-3]. The diagnosis of RLS is based on history with standardized clinical criteria, defined by the International RLS Study Group (IRLSSG) [4]. RLS is defined in two categories: idiopathic RLS (iRLS), which refers to familial tendency and no-known background condition while secondary RLS refers to various co morbid conditions, such as iron deficiency, diabetes mellitus, pregnancy, multiple sclerosis (MS), Parkinson's disease and end stage renal disease (ESRD) [5-12]. Prevalence of RLS in patients undergoing dialysis therapy due to ESRD has a wide range between $6.6 \%$ and $83 \%$ [13-24] depending on the varying diagnostic criteria applied in those studies, before the final standardization by the IRLSSG criteria. Moreover, this variation has been explained by the improvement of dialytic techniques as well as the pharmacological treatment of the ESRD.

The pathophysiological mechanisms involved in the idiopathic RLS are multifactorial, including dopaminergic dysfunction, spinal cord disinhibiting and dysfunction in the iron metabolism [25-29]. Regarding the secondary RLS in ESRD, iron-vitamin B-folic acid deficiency, $\mathrm{DM}$ as well as inadequate peritoneal dialysis (PD) was suggested to be the main risk factors for this condition.

Most of previous prevalence studies among ESRD patients have been conducted in those undergoing hemodialysis (HD) therapy and the results were mainly explained by the known risk factors as described above. To 
date, only a few studies addressed the prevalence of RLS among ESRD patients undergoing PD. Moreover, the real prevalence of RLS in ESRD, after excluding the patients with other known risk factors, is still unknown.

The aim of the current study was to evaluate the prevalence of RLS in ESRD patients treated with PD without known risk factors and to compare the clinical and biochemical characteristics of RLS patients with subjects without this condition.

\section{Methods}

\subsection{Patients}

The study was performed on 200 patients who were on PD treatments due to ESRD for at least 6 months and were followed up by Nephrology Department of Erciyes University Medical Faculty. Patients were also evaluated for RLS in Sleep Disorders Unit of Neurology Department of Erciyes University Medical Faculty. The local ethics committee approved the study protocol. The study was conducted in accordance with the declaration of Helsinki and local laws depending on whichever afforded greater protection to the patients. All the patients gave their informed consent of participation in the study.

We excluded 144 patients from the study who are under 18 years, or have either diabetes mellitus, anemia which was defined as a hemoglobin level $<10 \mathrm{~g} / \mathrm{dL}$, iron deficiency which was defined as a transferrin saturation $<20 \%$ and a serum ferritin concentration $<100 \mathrm{ng} / \mathrm{mL}$, Multiple Sclerosis, Parkinson's disease, pregnancy and/ or acute peritonitis because these conditions are important risk factors for symptomatic RLS. Also, patients who have taken medications such as antidepressants, neuroleptics or hypnotics that are known to affect sleep structure and have the sensorimotor symptoms of RLS were excluded.

Finally, 56 patients who fulfilled the inclusion and exclusion criteria were included in the study.

\subsection{Data Collection}

Firstly, clinical and laboratorial data of 56 patients were collected by the nephrologist of the PD unit. These data contained age, gender, body mass index, etiology of ESRD, smoking, drinking alcohol, use of erythropoietin, use of B complex vitamins and folic acid, duration of peritoneal dialysis, weekly Kt/Vurea, amount of residual urine volume, hemoglobin, serum calcium level, serum phosphorus level, calcium $\times$ phosphorus product, serum albumin level, serum iron level, total iron binding capacity, transferrin saturation, serum ferritin level, serum vitamin B12 level and serum folic acid level.

Secondly, all patients were examined by neurologist who were experts in sleep disorders and were evaluated with regard to presence of four essential RLS criteria as defined by the IRLSSG.

\subsection{Statistical Analysis}

SPSS 15.0 statistic software was used for the statistical analysis. The Kolmogorov-Smirnov test was used to determine the normality of the distributions of the variables. Continuous variables with normal distribution were presented as mean \pm standard deviation. Median value was used when normal distribution was absent. Statistical analysis for the parametric variables was performed by the Student's t test between two groups. The MannWhitney $U$ test was used to compare nonparametric variables between two groups. The qualitative variables were given as percent and the chi-square test and Fisher's exact test were used to compare variables between the two groups. $p$ value of $<0.05$ was considered to be significant.

\section{Results}

The mean age of the 56 patients was $47.10 \pm 12.42$ years. Thirty one of the 56 patients were male and the rest of them were female. RLS was found in $13(23.2 \%)$ of 56 PD patients. The most frequent cause of the etiology of end-stage renal disease was hypertension. The most of the patients had average peritoneal transport characteristics. The demographic, clinical, and biochemical parameters of the patients are summarized in Table 1.

Demographic, clinical, and biochemical parameters of the 13 patients with RLS are compared to 43 patients without RLS in Table 2. The use of vitamin B complex + folic acid supplements was significantly lower in patients with RLS than in those without RLS (69.2\% vs 97.7\%; p $=0.008)$. Although there was no statistically significant difference between two groups, folic acid levels were found in lower levels in patients with RLS than in those without RLS. On the other hand, there was no significant difference between patients with RLS and those without RLS in regard to age, gender, body mass index, cause of ESRD, peritoneal membrane transport characteristic, smoking, drinking, use of erythropoietin, duration of PD, hemoglobin, serum calcium, serum phosphorus, serum albumin, levels of serum iron, total iron binding capacity, ferritin, and vitamin B12, transferrin saturation, weekly $\mathrm{Kt} /$ Vurea value, and amount of residual urine volume ( $\mathrm{p}$ $>0.05)$.

\section{Discussion}

The prevalence of RLS in dialysis populations appears to be greater than in the general population although there is a wide variation, ranging between $6.6 \%$ and $83 \%$ [13-24]. Also, in previous studies which most of them were made in HD patients, no significant difference has been found between HD and PD patients in terms of RLS prevalence 
Table 1. Demographic, clinical and biochemical characteristics of patients on peritoneal dialysis.

\begin{tabular}{|c|c|}
\hline Age (year) & $47.10 \pm 12.42$ \\
\hline \multicolumn{2}{|l|}{ Gender } \\
\hline Male & $31(55.4 \%)$ \\
\hline Female & $25(44.6 \%)$ \\
\hline Body mass index $\left(\mathrm{kg} / \mathrm{m}^{2}\right)$ & $24.20 \pm 3.96$ \\
\hline \multicolumn{2}{|l|}{ Etiology of ESRD } \\
\hline Hypertension & $28(50.0 \%)$ \\
\hline Glomerulonephritis & $4(7.1 \%)$ \\
\hline Polycystic kidney disease & $3(5.4 \%)$ \\
\hline Amyloidosis & $2(3.6 \%)$ \\
\hline Obstructive nephropathy & $4(7.1 \%)$ \\
\hline Others & $1(1.8 \%)$ \\
\hline Unknown & $14(25 \%)$ \\
\hline \multicolumn{2}{|l|}{ Solute transport group according to PET } \\
\hline High & $7(12.5 \%)$ \\
\hline High-average & $19(33.9 \%)$ \\
\hline Low-average & $28(50 \%)$ \\
\hline Low & $2(3.6 \%)$ \\
\hline Smoking & $7(12.5 \%)$ \\
\hline Drinking alcohol & $2(3.6 \%)$ \\
\hline Use of erythropoietin & $33(58.9 \%)$ \\
\hline Use of B complex vitamins + folic acid & $51(91.1 \%)$ \\
\hline Duration of peritoneal dialysis (month) & $39.5(3-165)$ \\
\hline Hemoglobin (g/dL) & $11.42 \pm 1.29$ \\
\hline Serum calcium (mg/dL) & $8.82 \pm 0.90$ \\
\hline Serum phosphorus (mg/dl) & $4.26 \pm 1.17$ \\
\hline Serum albumin level (g/dL) & $3.41 \pm 0.38$ \\
\hline Iron $(\mu \mathrm{g} / \mathrm{dL})$ & $75.92 \pm 23.67$ \\
\hline Total iron binding capacity $(\mu \mathrm{g} / \mathrm{dL})$ & $243.21 \pm 49.53$ \\
\hline Transferrin saturation (\%) & $31.44 \pm 10.19$ \\
\hline Ferritin (ng/mL) & $335(107-2354)$ \\
\hline Vitamin B12 (pg/mL) & $546(89-1500)$ \\
\hline Folic acid (ng/mL) & $24(1-24)$ \\
\hline Weekly Kt/Vurea & $2.59 \pm 0.93$ \\
\hline Amount of residual urine volume (mL/day) & $500(0-2200)$ \\
\hline
\end{tabular}

ESRD: end-stage renal disease, PET: peritoneal equilibration test.
[13,17,30-32]. Our study group was constituted from under PD patients and prevalence of RLS was found 23.2 $\%$ when the other risk factors except for ESRD for secondary RLS were excluded. To date, only few studies evaluated the prevalence of RLS in PD patients [17,33, $34]$ and in those studies, RLS prevalence has been found between $19.7 \%$ [33] and $69.2 \%$ [34]. In the whole prevalence studies in uremic patients, RLS has been found more common than general population. This condition has been tried to be explained by increased frequency in presence of some risk factors such as iron deficiency, DM, vitamin and folic acid deficiencies in uremic patients. In present study, we studied the prevalence of RLS in ESRD patients treated with PD after risk factors that have been known to be frequently related with RLS were excluded. Consequently, prevalence of RLS in PD patients was found more common than general population even PD patients were applied to our exclusion criteria which are very strict to exclude any other possible factors that could be cause of secondary RLS. Although it is believed that RLS in PD patients evolve with different mechanism than RLS in general population, it is not clear what risk factors of RLS in PD patients are apart from known risk factors.

Although RLS pathophysiology are tried to be explained by different mechanism such as dopaminergic dysfunction, spinal disinhibiting and iron metabolism, it is still unclear. Also, pathophysiology of RLS in ESRD patients who are treated both HD and PD is unknown. One of mechanisms that are the most emphasized is anemia and iron deficiency. In fact, controversial results have been reported on the relationship between lower serum hemoglobin, ferritin, iron and RLS in ESRD patients, showing both the absence $[16,19,30,35]$ and presence of this association $[23,36]$. Especially, more recent studies have failed to show this association, but this might be caused by the routine use of high-dose intravenous iron and erythropoietin in modern dialysis management [29]. In current study, no significant differences were found in hemoglobin level, iron and ferritin levels in serum, total iron binding capacity, transferrin saturation and use of erythropoietin between PD patients with and without RLS.

Although prevalence of RLS has been reported to be higher in female gender and advanced age in general population, relationship between presence of RLS and age, gender in ESRD patients has not been exactly known. Some authors [23] have suggested that RLS prevalence in uremic patients is more in female gender and related with advanced age whereas others [16] and the current study haven't shown this relation.

Relationship between smoking, alcohol consumption and RLS in ESRD patients is controversial. Some studies suggest negatively effect of smoking and alcohol consumption 
Table 2. Comparison of demographic, clinical, and biochemical parameters between patients with RLS and those without RLS.

\begin{tabular}{|c|c|c|c|}
\hline Characteristic & RLS group (n: 13) & Non-RLS group (n: 43) & $\mathrm{p}$ value \\
\hline Age (year) & $48.00 \pm 11.95$ & $46.83 \pm 12.69$ & 0.771 \\
\hline Gender & & & 0.140 \\
\hline Female & $8(61.5 \%)$ & $17(39.5 \%)$ & \\
\hline Male & $5(38.5 \%)$ & $26(60.5 \%)$ & \\
\hline Body mass index $\left(\mathrm{kg} / \mathrm{m}^{2}\right)$ & $24.37 \pm 3.00$ & $24.14 \pm 4.24$ & 0.855 \\
\hline Etiology & & & 0.270 \\
\hline Hypertension & $7(53.8 \%)$ & $21(48.8 \%)$ & \\
\hline Glomerulonephritis & $2(15.4 \%)$ & $2(4.7 \%)$ & \\
\hline Polycystic kidney disease & $1(7.7 \%)$ & $2(4.7 \%)$ & \\
\hline Amyloidosis & - & $2(4.7 \%)$ & \\
\hline Obstructive nephropathy & - & $4(9.3 \%)$ & \\
\hline Others & $1(7.7 \%)$ & - & \\
\hline Unknown & $2(15.4 \%)$ & $12(27.9 \%)$ & \\
\hline Solute transport group according to PET & & & 0.252 \\
\hline High & $1(7.7 \%)$ & $6(14.0 \%)$ & \\
\hline High-average & $2(15.4 \%)$ & $17(39.5 \%)$ & \\
\hline Low-average & $9(69.2 \%)$ & $19(44.2 \%)$ & \\
\hline Low & $1(7.7 \%)$ & $1(2.3 \%)$ & \\
\hline Smoking & $2(15.4 \%)$ & $5(11.6 \%)$ & 0.519 \\
\hline Drinking alcohol & - & $2(4.7 \%)$ & 0.586 \\
\hline Use of erythropoietin & $8(61.5 \%)$ & $25(58.1 \%)$ & 0.545 \\
\hline Use of B complex vitamins + folic acid & $9(69.2 \%)$ & $42(97.7 \%)$ & 0.008 \\
\hline Duration of peritoneal dialysis (month) & $38(3-165)$ & $40(6-144)$ & 0.930 \\
\hline Hemoglobin (g/dL) & $11.80 \pm 1.44$ & $11.30 \pm 1.23$ & 0.221 \\
\hline Serum calcium $(\mathrm{mg} / \mathrm{dL})$ & $9.23 \pm 1.13$ & $8.70 \pm 0.79$ & 0.062 \\
\hline Serum phosphorus (mg/dl) & $4.61 \pm 1.44$ & $4.15 \pm 1.08$ & 0.219 \\
\hline Serum albumin level (g/dL) & $3.48 \pm 0.28$ & $3.38 \pm 0.40$ & 0.455 \\
\hline Iron $(\mu \mathrm{g} / \mathrm{dL})$ & $72.61 \pm 27.90$ & $75.76 \pm 23.36$ & 0.685 \\
\hline Total iron binding capacity $(\mu \mathrm{g} / \mathrm{dL})$ & $257.00 \pm 45.30$ & $239.04 \pm 50.49$ & 0.256 \\
\hline Transferrin saturation (\%) & $27.92 \pm 8.09$ & $32.24 \pm 10.91$ & 0.193 \\
\hline Ferritin (ng/mL) & $160(126-862)$ & $337.5(107-2354)$ & 0.188 \\
\hline Vitamin B12 (pg/mL) & $553(265-1000)$ & $546(89-1500)$ & 0.783 \\
\hline Folic acid $(\mathrm{ng} / \mathrm{mL})$ & $20(3-24)$ & $24(1-24)$ & 0.070 \\
\hline Weekly Kt/Vurea & $2.51 \pm 0.71$ & $2.61 \pm 1.00$ & 0.744 \\
\hline Amount of residual urine volume (mL/day) & $500(0-2000)$ & $500(0-2200)$ & 0.549 \\
\hline
\end{tabular}

RLS: Restless legs syndrome, PET: peritoneal equilibration test. 
on RLS in ESRD patients [37] whereas the others and our study have not demonstrated this relation $[30,38,39]$.

The role of serum calcium, phosphorus and intact parathormone levels in uremic RLS are controversial. Until today, relationship of RLS with high calcium [40], abnormal phosphorus [30], low [16] and high levels of PTH [21] have been described in uremic RLS patients. Moreover an anecdotal improvement of symptoms after parathyroidectomy have been described in uremic RLS [41].

On the other hand, there are also studies that lacked to show any significant difference on calcium, phosphorus and PTH levels [19,37]. In this study, no significant difference was found between ESRD patients with and without RLS in serum calcium, phosphorus and PTH levels.

It is not known which factors associated with dialysis play a role in the development of RLS in ESRD patients. For example, there are contradictory study results assessing affect of dialysis duration on presence of RLS $[16,21,42]$. The other dialysis parameter that is thought related to RLS is level of urea $\mathrm{Kt} / \mathrm{V}$. Urea $\mathrm{Kt} / \mathrm{V}$ is accepted as the optimal method for assessing the adequacy of dialysis. Some studies have suggested that RLS might represent a marker of inadequate dialysis in ESRD patients [37,43]. However, results of the present study showed any correlation neither dialysis duration nor level of $\mathrm{Kt} / \mathrm{v}$ urea between PD patients with and without RLS, concordant with some previous studies $[32,44]$.

Although in previous studies it was shown that vitamin B12 and folic acid are associated with RLS, our study did not confirm that in PD patients with RLS. However, in this study RLS was found to be more common in patients who are not taking B complex vitamins + folic acid supplement.

This study is the first and unique study that evaluates the prevalence of RLS on PD patients and describes clinical and biochemical parameters which may be related to RLS in patients under PD due to ESRD in Turkish patients. In fact the most important aspect of the study is that a prevalence study after other risk factors for RLS are excluded.

RLS is a very important sleep related sensorimotor disorder that decreases life quality and increases morbidity and mortality rates in PD patients. Therefore RLS must be diagnosed and treated at early period. Also, vitamin B and folic acid supplements could be used to decrease RLS prevalence in PD patients.

\section{REFERENCES}

[1] K. A. Ekbom, "Restless Legs Syndrome," Neurology, Vol. 10, 1960, pp. 868-873. doi:10.1212/WNL.10.9.868

[2] G. J. Lavigne and J. Y. Montplaisir, "Restless Legs Syn- drome and Sleep Bruxism: Prevalence and Association among Canadians," Sleep, Vol. 17, No. 8, 1994, pp. 739743.

[3] B. Phillips, T. Young, L. Finn, et al., "Epidemiology of Restless Legs Symptoms in Adults," Archives of Internal Medicine, Vol. 160, No. 14, 2000, pp. 2137-2141. doi:10.1001/archinte.160.14.2137

[4] R. P. Allen, D. Picchietti, W. A. Hening, et al., "Restless Legs Syndrome: Diagnostic Criteria, Special Considerations and Epidemiology. A Report from the Restless Legs Syndrome Diagnosis and Epidemiology Workshop at the National Institutes of Health," Sleep Medicine, Vol. 4, No. 2, 2003, pp. 101-119.

[5] M. Zucconi and L. Ferini-Strambi, "Epidemiology and Clinical Findings of Restless Legs Syndrome," Sleep Medicine, Vol. 5, No. 3, 2004, pp. 293-299. doi:10.1016/j.sleep.2004.01.004

[6] R. P. Allen and C. J. Earley, "Restless Legs Syndrome: A Review of Clinical and Pathophysiologic Features," Journal of Clinical Neurophysiology, Vol. 18, No. 2, 2001, pp. 128-147. doi:10.1097/00004691-200103000-00004

[7] L. A. Lopes, C. M. Lins, V. G. Adeodato, et al., "Restless Legs Syndrome and Quality of Sleep in Type 2 Diabetes," Diabetes Care, Vol. 28, No. 11, 2005, pp. 2633-2636. doi:10.2337/diacare.28.11.2633

[8] M. Manconi, V. Govoni, A. De Vito, et al., "Pregnancy as a Risk Factor for Restless Legs Syndrome," Sleep Medicine, Vol. 5, No. 3, 2004, pp. 305-308. doi:10.1016/j.sleep.2004.01.013

[9] B. Phillips, T. Young, L. Finn, et al., "Epidemiology of Restless Legs Symptoms in Adults," Archives of Internal Medicine, Vol. 160, No. 14, 2000, pp. 2137-2141. doi:10.1001/archinte.160.14.2137

[10] N. C. Moreira, R. S. Damasceno, C. A. Medeiros, et al., "Restless Leg Syndrome, Sleep Quality and Fatigue in Multiple Sclerosis Patients," Brazilian Journal of Medical and Biological Research, Vol. 41, No. 10, 2008, pp. $932-$ 937. doi:10.1590/S0100-879X2008001000017

[11] T. Nomura, Y. Inoue, M. Miyake, et al., "Prevalence and Clinical Characteristics of Restless Legs Syndrome in Japanese Patients with Parkinson's Disease," Movement Disorders, Vol. 21, No. 3, 2006, pp. 380-384. doi: $10.1002 / \mathrm{mds} .20734$

[12] J. P. Bastos, R. B. Sousa, L. A. Nepomuceno, et al., "Sleep Disturbances in Patients on Maintenance Hemodialysis: Role of Dialysis Shift," Revista da Associação Médica Brasileira, Vol. 53, No. 6, 2007, pp. 492-496. doi:10.1590/S0104-42302007000600014

[13] J. L. Holley, S. Nespor and R. Rault, "A Comparison of Reported Sleep Disorders in Patients on Chronic Hemodialysis and Continuous Peritoneal Dialysis," American Journal of Kidney Diseases, Vol. 2, 1992, pp. 156-161.

[14] S. L. Walker, A. Fine and M. H. Kryger, "Sleep Complaints Are Common in Dialysis Unit," American Journal of Kidney Diseases, Vol. 26, No. 5, 1995, pp. 751-756. doi:10.1016/0272-6386(95)90438-7

[15] J. W. Winkelman, G. M. Chertow and J. M. Lazarus, "Restless Legs Syndrome in End-Stage Renal Disease," 
American Journal of Kidney Diseases, Vol. 28, No. 3, 1996, pp. 372-378. doi:10.1016/S0272-6386(96)90494-1

[16] V. Collado-Seidel, R. Kohnen, W. Samtleben, et al., "Clinical and Biochemical Findings in Uremic Patients with and without Restless Legs Syndrome," American Journal of Kidney Diseases, Vol. 31, No. 2, 1998, pp. 324-328. doi:10.1053/ajkd.1998.v31.pm9469505

[17] D. S. C. Hui, T. Y. H. Wong, F. W. S. Ko, et al., "Prevalence of Sleep Disturbances in Chinese Patients with EndStage Renal Failure on Continuous Ambulatory Peritoneal Dialysis," American Journal of Kidney Diseases, Vol. 36, No. 4, 2000, pp. 783-788. doi:10.1053/ajkd.2000.17664

[18] Q. Huiqi, L. Shan and Q. Mingcai, "Restless Legs Syndrome (RLS) in Uremic Patients Is Related to the Frequency of Hemodialysis Sessions," Nephron, Vol. 86, No. 4, 2000, p. 540. doi:10.1159/000045861

[19] M. Miranda, F. Araya, J. L. Castillo, et al., "Restless Legs Syndrome: A Clinical Study in Chilean General Population and in Uremic Patients," Revista Médica de Chile, Vol. 129, 2001, pp. 179-186.

[20] M. L. Thorp, C. D. Morris and S. P. Bugby, "A Crossover Study of Gabapentin in Treatment of Restless Legs Syndrome among Hemodialysis Patients," American Journal of Kidney Diseases, Vol. 38, No. 1, 2001, pp. 104-108. doi:10.1053/ajkd.2001.25202

[21] M. Sabbatini, B. Minale, A. Crispo, et al., "Insomnia in Maintenance Haemodialysis Patients," Nephrology Dialysis Transplantation, Vol. 17, No. 5, 2002, pp. 852-856. doi:10.1093/ndt/17.5.852

[22] D. S. C. Hui, T. Y. H. Wong, T. S. T. Li, et al., "Prevalence of Sleep Disturbances in Chinese Patients with End Stage Renal Failure on Maintenenance Hemodialysis," Medical Science Monitor, Vol. 8, No. 5, 2002, pp. 331336.

[23] J. Takaki, T. Nishi, M. Nangaku, et al., "Clinical and Psychological Aspects of Restless Legs Syndrome in Uremic Patients on Hemodialysis," American Journal of Kidney Diseases, Vol. 41, No. 4, 2003, pp. 833-839. doi:10.1016/S0272-6386(03)00031-3

[24] D. Bhowmik, M. Bhatia, S. Gupta, et al., "Restless Legs Syndrome in Hemodialysis Patients in India: A Case Controlled Study," Sleep Medicine, Vol. 4, No. 2, 2003, pp. 143-146. doi:10.1016/S1389-9457(03)00005-4

[25] S. Akpinar, "Treatment of Restless Legs with Levodopa plus Benserazide," Archives of Neurology, Vol. 39, No. 11, 1982, p. 739. doi:10.1001/archneur.1982.00510230065027

[26] J. Montplaisir, R. Godbout, G. Poirer, et al., "Restless Legs Syndrome and Periodic Movements in Sleep; Physiology and Treatment with L-Dopa," Clinical Neuropharmacology, Vol. 9, No. 5, 1986, pp. 456-463. doi:10.1097/00002826-198610000-00006

[27] J. Winkelmann, J. Schadrack, T. Wetter, et al., "Opioid and Dopamine Antagonist Drug Challenges in Untreated Restless Legs Syndrome Patients," Sleep Medicine, Vol. 2, No. 1, 2001, pp. 57-61. doi:10.1016/S1389-9457(00)00025-3
[28] M. Aksu and W. Bara-Jimenez, "State Dependent Excitability Changes of Spinal Flexor Reflex in Patients with Restless Legs Syndrome Secondary to Chronic Renal Failure," Sleep Medicine, Vol. 2, No. 1, 2002, pp. $427-$ 430. doi:10.1016/S1389-9457(02)00073-4

[29] R. L. Benz, M. R. Pressman, E. T. Hovick, et al., "A Preliminary Study of the Effects of Correction of Anemia with Recombinant Human Erythropoietin Therapy on Sleep, Sleep Disorders, and Daytime Sleepiness in Hemodialysis Patients (The SLEEPO Study)," American Journal of Kidney Diseases, Vol. 34, No. 6, 1999, pp. 10891095. doi:10.1016/S0272-6386(99)70015-6

[30] G. L. Gigli, M. Adorati, P. Dolso, et al., "Restless Legs Syndrome in End-Stage Renal Disease," Sleep Medicine, Vol. 5, No. 3, 2004, pp. 309-315. doi:10.1016/j.sleep.2004.01.014

[31] N. G. Kutner, R. Zhang, Y. Huang, et al., "Racial Differences in Restless Legs Symptoms and Serum Ferritin in an Incident Dialysis Patient Cohort," International Urology and Nephrology, Vol. 44, No. 6, 2012, pp. 18251831.

[32] G. Merlino, S. Lorenzut, G. Romano, et al., "Restless Legs Syndrome in Dialysis Patients: A Comparison between Hemodialysis and Continuous Ambulatory Peritoneal Dialysis," Neurological Sciences, Vol. 33, No. 6, 2012, pp. 1311-1318.

[33] M. L. Unruh, A. S. Levey, C. D’Ambrosio, et al., "Restless Legs Symptoms among Incident Dialysis Patients: Association with Lower Quality of Life and Shorter Survival. Choices for Healthy Outcomes in Caring for EndStage Renal Disease (CHOICE) Study," American Journal of Kidney Diseases, Vol. 43, No. 5, 2004, pp. 900909. doi:10.1053/j.ajkd.2004.01.013

[34] H. H. Al-Jahdali, W. A. Al-Qadhi, H. A. Khogeer, et al., "Restless Legs Syndrome in Patients on Dialysis," Saudi Journal of Kidney Diseases and Transplantation, Vol. 20, No. 3, 2009, pp. 378-385.

[35] S. Siddiqui, M. Mak, D. Kavanagh, et al., "Restless Legs Syndrome in Haemodialysis Patients," Scottish Renal Association, 9-10 May 2003.

[36] S. Roger, D. Harris and J. Stewart, "Possible Relation between Restless Legs and Anemia in Renal Dialysis Patients," Lancet, Vol. 337, No. 8756, 1991, p. 1551. doi:10.1016/0140-6736(91)93248-8

[37] W. C. Chen, P. S. Lim, W. C. Wu, et al., "Sleep Behavior Disorders in a Large Cohort of Chinese (Taiwanese) Patients Maintained by Long-Term Hemodialysis," American Journal of Kidney Diseases, Vol. 48, No. 2, 2006, pp. 277-284. doi:10.1053/j.ajkd.2006.04.079

[38] S. M. Araujo, V. M. de Bruin, L. A. Nepomuceno, et al., "Restless Legs Syndrome in End-Stage Renal Disease: Clinical Characteristics and Associated Comorbidities," Sleep Medicine, Vol. 11, No. 8, 2010, pp. 785-790. doi:10.1016/j.sleep.2010.02.011

[39] C. Quinn, M. Uzbeck, I. Saleem, et al., "Iron Status and Chronic Kidney Disease Predict Restless Legs Syndrome in an Older Hospital Population," Sleep Medicine, Vol. 12, No. 3, 2011, pp. 295-301. doi:10.1016/j.sleep.2010.08.014 
[40] A. Kawauchi, Y. Inoue, T. Hashimoto, et al., "Restless Legs Syndrome in Hemodialysis Patients: Health-Related Quality of Life and Laboratory Data Analysis," Clinical Nephrology, Vol. 66, No. 6, 2006, pp. 440-446.

[41] L. L. Lim, D. Dinner, K. W. Tham, et al., "Restless Legs Syndrome Associated with Primary Hyperparathyroidism," Sleep Medicine, Vol. 6, No. 3, 2005, pp. 283-285. doi:10.1016/j.sleep.2004.10.014

[42] N. Kutner and D. Bliwise, "Restless Legs Complaint in African-American and Caucasian Hemodialysis Patients,"
Sleep Medicine, Vol. 3, No. 6, 2002, pp. 497-500. doi:10.1016/S1389-9457(02)00025-4

[43] J. Perl, M. L. Unruh and C. T. Chan, "Sleep Disorders in End-Stage Renal Disease: 'Markers of Inadequate Dialysis'?" Kidney International, Vol. 70, 2006, pp. $1687-$ 1693. doi:10.1038/sj.ki.5001791

[44] J. L. Holley, S. Nespor and R. Rault, "Characterizing Sleep Disorders in Chronic Hemodialysis Patients," ASAIO Transactions, Vol. 37, No. 3, 1991, pp. M456-M457. 